\title{
Pulsating variables in NGC 3293, the open cluster with the most $\beta$ Cephei stars known
}

\author{
G. Handler, ${ }^{1}$ T. Tuvikene, ${ }^{2}$ D. Lorenz, ${ }^{1}$ S. Saesen, ${ }^{3}$ J. L. Provencal, ${ }^{4}$ R. R. Shobbrook,,${ }^{5}$ \\ M. Pagani, ${ }^{6}$ B. Quint, ${ }^{6}$ M. Desmet,${ }^{3}$ C. Sterken, ${ }^{2}$ A. Kanaan, ${ }^{6}$ C. Aerts ${ }^{3,7}$ \\ ${ }^{1}$ Institute of Astronomy, University of Vienna, Austria \\ ${ }^{2}$ Vrije Universiteit Brussel, Belgium \\ ${ }^{3}$ Instituut voor Sterrenkunde, K. U. Leuven, Belgium \\ ${ }^{4}$ University of Delaware and Mt. Cuba Observatory, USA \\ ${ }^{5}$ Research School of Astronomy and Astrophysics, Australian National University \\ ${ }^{6}$ Universidade Federal de Santa Catarina, Brazil \\ ${ }^{7}$ Department of Astrophysics, Radboud University Nijmegen, The Netherlands
}

\begin{abstract}
We carried out an extensive CCD photometry campaign of the open cluster NGC 3293 that contains eleven known $\beta$ Cephei stars. Preliminary results indicate that none of these is singly periodic. Several objects are among the most multiperiodic of these massive pulsators, giving us strong hope to perform precision asteroseismology in an open cluster for the first time.

We also report a peculiar group of variables in NGC 3293 that is located near the lowluminosity end of the $\beta$ Cephei instability strip. The variability periods of these stars are too long for classical $\beta$ Cephei pulsation, but too short for binarity or rotational effects, or for SPB-type pulsation. In addition, we discovered about a dozen $\delta$ Scuti stars in the field.
\end{abstract}

\section{Introduction}

Pigulski (2007) recently reviewed the initial successes of asteroseismology of $\beta$ Cephei stars, where first constraints on interior stellar structure such as differential rotation, were obtained. As only stars in the centre of the $\beta$ Cephei instability strip could be investigated so far, the asteroseismically available parameter space in the HR diagram needs to be extended, i.e. stars of lower and higher mass are to be examined. The open cluster NGC 3293 is ideal for this purpose: it contains eleven known $\beta$ Cephei stars, many of which belong to the most massive representatives of the group. In addition, other advantages of open cluster asteroseismology (assumption of same metallicity, many targets in the same field) can be exploited.

Therefore, we carried out a massive CCD photometry campaign of NGC 3293, involving six sites in the Southern Hemisphere. More than 700 hours of high-quality UBV photometry were obtained from January to May, 2006.

\section{First results}

From a frequency analysis of the 32 nights of $U$ data collected by the first author at the South African Astronomical Observatory (SAAO), we find that all ten $\beta$ Cephei stars in the centre of the cluster are multiperiodic, with a maximum of five frequencies (V378 Car) detected in these data so far. Several stars, including the eclipsing binary V381 Car, show four pulsation frequencies in these data. V406 Car exhibits an equally spaced frequency triplet. Given the large amount of data we collected, we expect that our final detection level for pulsation modes will be better than $0.5 \mathrm{mmag}$. Combining this with the expected clean spectral window due to our multisite coverage, we are optimistic that our observational results for the $\beta$ Cephei stars will enable detailed asteroseismic modelling. 


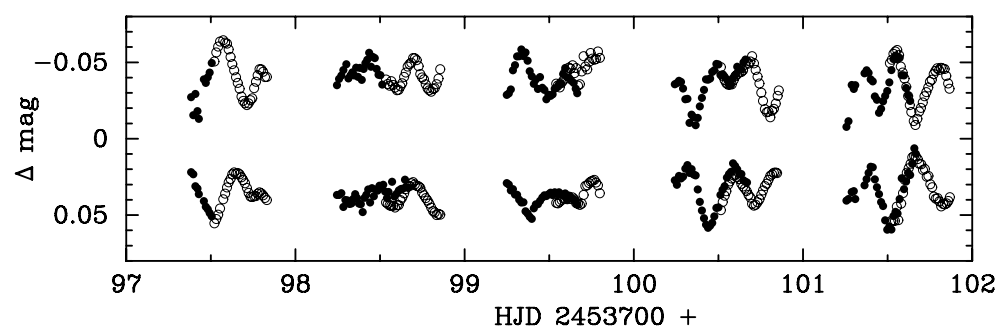

Figure 1: Light curves of the two mid B-type variables NSV 18451 (upper curve) and NSV 18457 (lower curve) from our campaign. The filled circles are SAAO data, the open circles are CTIO measurements. Complicated variability is evident.

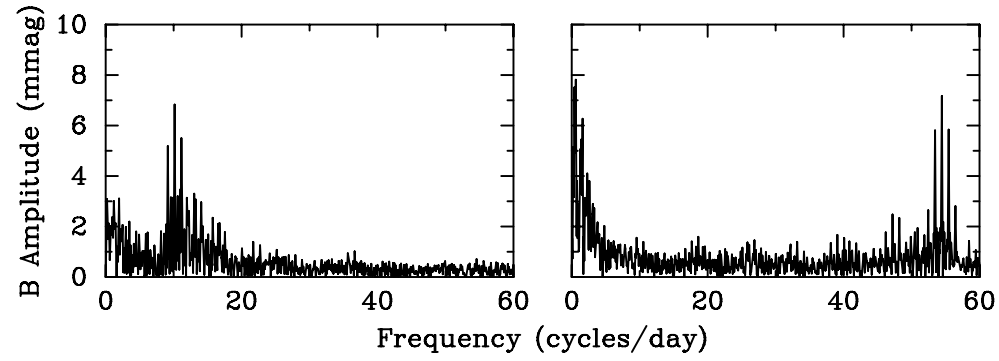

Figure 2: Amplitude spectra of the two $\delta$ Scuti stars BVC 183 (left panel) and BVC 363 (right panel) in the field of NGC 3293 (B data from CTIO). The identification numbers are from Baume et al. (2003).

There is a second group of B-type variables in this cluster. These stars are $1-2$ magnitudes fainter than the $\beta$ Cephei stars, their variability occurs on longer time scales ( $\sim 8$ to 12 $\mathrm{hr}$ ), and often seems multiperiodic (see Fig. 1 for some examples). The variability time scales of these stars is shorter than those of known SPB stars, according to the on-line compilation of De Cat (2004), in most cases shorter than the critical rotation period of mid B stars (about 10 hours), but longer than the time scale expected for $\beta$ Cephei-type pulsation (see Stankov \& Handler 2005). The variability of these stars may be connected to their rapid rotation.

In the fainter part of the HR diagram of NGC 3293, one would expect the presence of $\delta$ Scuti stars. Indeed, we found over a dozen candidates of this type of variables; some of them are foreground objects, however. Amplitude spectra of two confirmed $\delta$ Scuti stars are shown in Fig. 2. The presence of both $\beta$ Cephei and $\delta$ Scuti stars in NGC 3293 may result in a useful test of stellar opacities (Pamyatnykh, private communication).

Acknowledgments. This work has been supported by the Austrian Fonds zur Förderung der wissenschaftlichen Forschung under grant P18339-N08.

\section{References}

Baume G., Vazquez R. A., Carraro G., Feinstein A., 2003, A\&A, 402, 549

De Cat P., 2004, http://www.ster.kuleuven.ac.be/ peter/Bstars/

Pigulski A., 2007, Comm. Asteroseis., 150, 159

Stankov A., Handler G., 2005, ApJS, 158, 193 